\title{
Factors influencing health behaviours in response to the air quality health index: a cross-sectional study in Hamilton, Canada
}

\author{
Sally Radisic* ${ }^{* \dagger}$, K. Bruce Newbold*, John Eyles** and Allison Williams* \\ *School of Geography and Earth Sciences, McMaster University, Hamilton, ON, Canada. \\ ${ }^{\dagger}$ City of Hamilton Public Health Services, Hamilton, ON, Canada. \\ *Centre for Health Policy, School of Public Health, University of Witwatersrand, Johannesburg, South Africa.
}

\begin{abstract}
Research associating adverse health effects with air pollution exposure is robust. Public health authorities recognize the need to implement population health strategies that protect public health from air pollution exposure. The Air Quality Health Index (AQHI) is a public health initiative that is intended to protect the public's health from exposure to air pollution. The aim of this research was to identify and explain factors influencing AQHI adoption at the individual level and to establish intervention strategies. A cross-sectional survey with both quantitative and qualitative questions was administered in Hamilton, Ontario, Canada, during the months of June to October 2012. Logistic regression and the Health Belief Model are used to explore the data. Demographics (gender, age, education, and area of residence), knowledge/understanding, and individual risk perceptions (neighbourhood air effects on health) were found to be significant predictors of $\mathrm{AQHI}$ adoption. The perceived benefits of $\mathrm{AQHI}$ adoption included protection of health for self and those cared for via familial and (or) occupational duties, whereas the perceived barriers of AQHI adoption included lack of knowledge about where to check and lack of time required to check and follow AQHI health messages. Also, self-efficacy was uncovered as a factor influencing AQHI adoption. Accordingly, increases in AQHI adoption could be achieved via increasing $A Q H I$ knowledge among low socioeconomic status females, communicating the benefits of $A Q H I$ adoption to "at-risk" populations and implementing supports for males to follow AQHI health messages.
\end{abstract}

Key words: public health, air pollution, Air Quality Health Index, health behaviour, Health Belief Model, socioeconomic status, environmental risk perceptions

\section{Introduction}

Air pollution is an environmental health issue receiving a great deal of attention because of the detrimental effects it has on population health (WHO, 2014). Research has consistently found adverse respiratory and cardiovascular health effects associated with air pollution exposure (Dockery et al., 1993; Pope et al., 2002; Zanobetti and Schwartz, 2009). Consequently, the World Health Organization (WHO) acknowledged outdoor air pollution as a human carcinogen (IARC and WHO, 2013).

Public health authorities recognize the need to implement population health strategies that protect public health from air pollution exposure. In Canada, the Air Quality Health Index (AQHI) is a health protection tool developed by the federal government (www.airhealth.ca) to provide air quality and health information such that the public can implement health protective behaviours (reducing and (or) rescheduling outdoor activity) and decrease exposure to outdoor air pollution (Environment Canada, 2013).

Corresponding author: Sally Radisic (email: sally.radisic@hamilton.ca)
The AQHI is a comparatively easy to understand 10-point scale (low risk $1-3$, medium risk $4-6$, high risk $7-10$, very high risk > 10) (Environment Canada, 2013). Unlike the Air Quality Index (AQI) that was based on six pollutants (ozone $\left(\mathrm{O}_{3}\right)$, fine particulate matter $\left(\mathrm{PM}_{2.5}\right)$, nitrogen dioxide $\left(\mathrm{NO}_{2}\right)$, sulphur dioxide $\left(\mathrm{SO}_{2}\right)$, carbon monoxide (CO), and total reduced sulphur (TRS)) and communicates the single worst pollutant, the AQHI communicates the cumulative health risks for the pollutants in the index $\left(\mathrm{O}_{3}, \mathrm{NO}_{2}, \mathrm{PM}_{2.5}\right)$ known to be hazardous to human health. Hence, the AQHI is considered best suited to communicate health risks associated with air pollution (Environment Canada, 2013). The AQHI provides health messages for each category of health risk advising the public to implement health protective behaviour for both the "at-risk" (young children, elderly, and those with pre-existing respiratory and (or) cardiovascular conditions) and general populations (Table 1). Therefore, adopting the AQHI as health protective behaviour would require an individual to: (i) be aware of AQHI, (ii) check AQHI numbers, and (iii) follow AQHI health messages.

As one of the original theories of health behaviour, the Health Belief Model (HBM) is also one of the most 
Table 1. Air Quality Health Index (AQHI) messages

\begin{tabular}{|l|c|l|l|}
\hline & & \multicolumn{1}{|c|}{ Health messages } \\
\hline Health risk & AQHI & \multicolumn{1}{|c|}{ At-risk population* } & \multicolumn{1}{|c|}{ General population } \\
\hline Low & $1-3$ & Enjoy your usual outdoor activities. & Ideal air quality for outdoor activities. \\
\hline Moderate & $4-6$ & $\begin{array}{l}\text { Consider reducing or rescheduling strenuous } \\
\text { activities outdoors if you are experiencing } \\
\text { symptoms. }\end{array}$ & $\begin{array}{l}\text { No need to modify your usual outdoor activities } \\
\text { unless you experience symptoms such as coughing } \\
\text { and throat irritation. }\end{array}$ \\
\hline High & $7-10$ & $\begin{array}{l}\text { Reduce or reschedule strenuous activities } \\
\text { outdoors. Children and the elderly should } \\
\text { also take it easy. }\end{array}$ & $\begin{array}{l}\text { Consider reducing or rescheduling strenuous } \\
\text { activities outdoors if you experience symptoms such } \\
\text { as coughing and throat irritation. }\end{array}$ \\
\hline Very high & $>10$ & $\begin{array}{l}\text { Avoid strenuous activities outdoors. Children } \\
\text { and the elderly should also avoid outdoor } \\
\text { physical exertion. }\end{array}$ & $\begin{array}{l}\text { Reduce or reschedule strenuous activities outdoors, } \\
\text { especially if you experience symptoms such as } \\
\text { coughing and throat irritation }\end{array}$ \\
\hline
\end{tabular}

*People with heart or breathing problems are at greater risk.

Source: Environment Canada, 2013.

extensively used to explain health behaviour (Glanz et al., 2008). Developed to address public health concerns in the 1950s, (Hochbaum, 1958; Rosenstock, 1974) the model consists of six constructs that explain why individuals will participate in behaviour designed to prevent adverse health effects, including: perceived susceptibility, perceived severity, perceived benefits, perceived barriers, cues to action, and self-efficacy. The model is intuitive in the sense that it claims if a person believes he/she is susceptible to an exposure (i.e., air pollution), believes that engaging in a course of action available to him/her (i.e., AQHI adoption) would be beneficial in reducing either susceptibility to or severity of the exposure, and believes that the benefits of engaging in the action (i.e., prevention of adverse health effects) prevail over the costs of action (i.e., time commitments checking AQHI), he/she will engage in the action that is considered to decrease his/her risk (Glanz et al., 2008). The model has also been effectively used to explain and guide public health intervention strategies including breast cancer screening (Legler et al., 2002), injury prevention (Trifiletti et al., 2005), and HIV/AIDS-linked behaviours (Noar et al., 2009). Thus, the $\mathrm{HBM}$ can be used to explain AQHI adoption and guide intervention strategies.

Many studies have documented the detrimental effects of air pollution on health, but Semenza et al. (2008) point out that there are few studies (Stieb et al., 1996) that examine the effects of air quality information on health behaviour. This study examines the effects of air quality information on health behaviour by determining what factors influence the adoption of the AQHI in Hamilton.

\section{Methods}

\section{Survey instrument}

The questionnaire developed for the study was based on the HBM constructs and individual characteristics identified as key components of environmental risk perception including: demographics, socioeconomic status (SES), and health status (Elliott et al., 1999; Eyles et al., 2009; Wilson et al., 2009; Simone et al.,
2012). The survey included both quantitative and qualitative questions to identify and explain AQHI: awareness, checking, following of health messages, and adoption. AQHI awareness was assessed with the survey question: "Have you heard of the Air Quality Health Index (AQHI)?” To assess if participants were checking AQHI, the question: "Do you check the Air Quality Health Index (AQHI)?" was asked. In addition, to assess if participants were following AQHI health messages, the survey asked: "Do you follow AQHI Health Messages which tell you when to consider reducing or re-scheduling outdoor physical activity?" Responses to all three questions (AQHI awareness, AQHI checking, AQHI following) were used together to establish AQHI adoption.

\section{Study area}

The city of Hamilton, Ontario, is an industrial city consisting of a population of over 519000 people, with $84.1 \%$ speaking English in the home (Statistics Canada, 2012a). Several studies have identified that there are spatial variations in air pollution concentrations in Hamilton (Jerrett et al., 2001; Buzzelli et al., 2003; Wallace et al., 2010). A number of factors contribute to the spatial variability of air pollution including: vehicles/traffic, industry/facilities, meteorological conditions/atmospheric inversions, and the geographical upper and lower city divide by the Niagara Escarpment, potentially entrapping pollutants (Wallace et al., 2010).

Hamilton has experienced a demographic shift with wealthier individuals moving out of the lower city and into the higher SES suburban areas; leaving those individuals of lower SES in the lower city (DeLuca et al., 2012). To determine if demographics, SES, and health status are influencing AQHI adoption, this study applied the same four quadrant division of the urban areas used previously by other researchers studying air pollution and health in Hamilton (Kerigan et al., 1986; Barakat-Haddad et al., 2013). The four urban areas include: east lower, west lower (including the merged industrial core), east upper, and west upper (Fig. 1). In addition to the four 
urban areas, this study includes five suburban areas: Ancaster, Dundas, Flamborough, Stoney Creek, and Glanbrook. Due to the low response rate, the suburban areas of Ancaster, Dundas, and Flamborough were combined to represent one suburban area for analysis purposes.

The use of the four quadrant urban area divisions along with the three additional suburban divisions accounts for spatial variations in air pollution (Wallace et al., 2010), demographic, socioeconomic, and health differences (DeLuca et al., 2012) that, according to the HBM, are linked to perceived threat (perceived susceptibility plus perceived severity), benefits, barriers, and self-efficacy. Therefore, the division of the city into seven distinct areas allows the HBM to explain and guide AQHI adoption in Hamilton.

\section{Sample}

Participants were recruited at fairs in the urban and suburban areas of Hamilton during the months of June to October 2012. An AQHI promotional booth was set up where participants had the opportunity to participate by completing a paper and pencil survey. Inclusion criteria included being a Hamilton resident and at least 18 years of age and older. The study consisted of a convenience sample of 707 participants who received AQHI promotional materials (i.e., water bottle, Frisbee, beach ball) as compensation for participating. This research received ethics approval from the McMaster University Research Ethics Board and informed consent was received from participants prior to conducting the study.

\section{Data analysis}

There were two broad phases to the analysis. First, logistic regression analysis using SPSS (version 22) was used to predict AQHI awareness, checking, following, and adoption from demographics, knowledge/understanding, individual perceptions, and pre-existing conditions. The four dichotomous (yes/no) dependent variables included: AQHI awareness, AQHI checking, AQHI following, and AQHI adoption.

The independent variables used in the logistic regression are outlined in Table 2 along with the survey questions and coded responses. The independent variables included: gender, age, household income, education, and area of residence. Moreover, AQHI knowledge/understanding was included to determine if there was a difference between being aware of the term and understanding what it means. In addition, individual perceptions including those about neighborhood air quality and physical environment impacts on health along with the length of time of these perceptions were incorporated. Other individual perceptions included the amount of time participants estimated they spent outdoors and how they perceived their

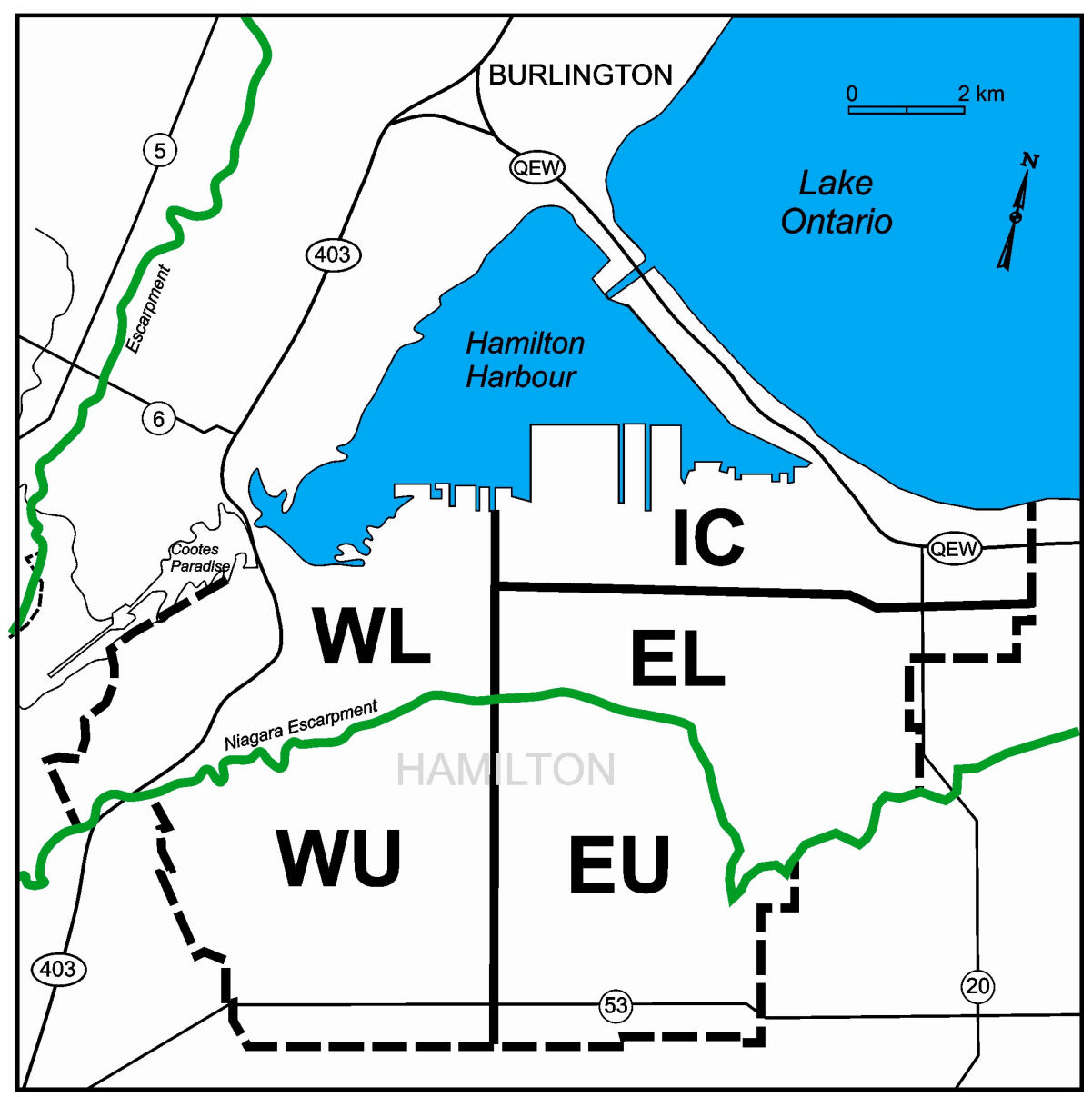

Figure 1. Division of urban areas. (EL, east lower; WL, west lower; IC, the merged industrial core; EU, east upper; and WU, west upper.) Source: Barakat-Haddad et al. (2013), Kerigan et al. (1986). 
Table 2. Independent variables and survey questions

\begin{tabular}{|c|c|c|}
\hline Independent variable & Survey question & Coded responses \\
\hline Gender & What is your sex? & $\begin{array}{l}\text { Male } \\
\text { Female }\end{array}$ \\
\hline Age & What is your age?(y) & $\begin{array}{l}18-24 \\
25-34 \\
35-44 \\
45-54 \\
55-64 \\
\geq 65 \text { and over }\end{array}$ \\
\hline Income & What is your household income? & $\begin{array}{l}<\$ 20000 \\
\$ 21000-\$ 50000 \\
\$ 51000-\$ 80000 \\
\geq \$ 81000\end{array}$ \\
\hline Education & What is the highest level of education you completed? & $\begin{array}{l}\text { Elementary School } \\
\text { High School } \\
\text { Trade } \\
\text { College } \\
\text { University }\end{array}$ \\
\hline Area of residence & $\begin{array}{l}\text { What is your postal code? } \\
\text { Where do you live? }\end{array}$ & $\begin{array}{l}\text { Ancaster } \\
\text { Dundas } \\
\text { Flamborough } \\
\text { Glanbrook } \\
\text { Hamilton } \\
\text { Stoney Creek } \\
\text { Other } \\
\text { Please specify }\end{array}$ \\
\hline AQHI Knowledge/understanding & Do you know what a High AQHI (7-10) means? & Yes No Not sure \\
\hline Know where to check & $\begin{array}{l}\text { Do you know where to check for daily Air Quality Health } \\
\text { Index (AQHI)? }\end{array}$ & Yes No Not sure \\
\hline $\begin{array}{l}\text { Perceived neighborhood air effects on } \\
\text { health }\end{array}$ & $\begin{array}{l}\text { Do you think the air in your neighbourhood affects your } \\
\text { health? }\end{array}$ & Yes No Not sure \\
\hline $\begin{array}{l}\text { Length of time perceive neighborhood } \\
\text { air effects on health }\end{array}$ & $\begin{array}{l}\text { How long have you felt this way about the air in your } \\
\text { neighbourhood? }\end{array}$ & $\begin{array}{l}\text { Last week } \\
\text { Last month } \\
\text { Last } 6 \text { months } \\
\text { Last year } \\
\text { Last } 5 \text { years } \\
\text { Last } 10 \text { years } \\
\text { Other } \\
\text { Please Specify }\end{array}$ \\
\hline $\begin{array}{l}\text { Perceived neighborhood physical } \\
\text { environment effects on health }\end{array}$ & $\begin{array}{l}\text { Do you think the physical environment (i.e., buildings, vehicles/ } \\
\text { traffic, trees, etc.) in your neighbourhood affects your health? }\end{array}$ & Yes No Not sure \\
\hline $\begin{array}{l}\text { Length of time perceive neighborhood } \\
\text { physical environment on health }\end{array}$ & $\begin{array}{l}\text { How long have you felt this way about the physical } \\
\text { environment in your neighbourhood? }\end{array}$ & $\begin{array}{l}\text { Last week } \\
\text { Last month } \\
\text { Last } 6 \text { months } \\
\text { Last year } \\
\text { Last } 5 \text { years } \\
\text { Last } 10 \text { years } \\
\text { Other } \\
\text { Please Specify }\end{array}$ \\
\hline
\end{tabular}


Table 2 (continued).

\begin{tabular}{|c|c|c|}
\hline Independent variable & Survey question & Coded responses \\
\hline Time spent outside & $\begin{array}{l}\text { How much of your time, in the summer, is spent outside doing } \\
\text { physical activity? }\end{array}$ & $\begin{array}{l}\text { Most of my time } \\
\text { Some of my time } \\
\text { Hardly any of my } \\
\text { time } \\
\text { None of my time }\end{array}$ \\
\hline Health status (self-reported) & How would you describe your current overall health? & $\begin{array}{l}\text { Very Good } \\
\text { Good } \\
\text { Fair } \\
\text { Poor } \\
\text { Very Poor }\end{array}$ \\
\hline Pre-existing respiratory condition & $\begin{array}{l}\text { Do you have any existing respiratory (breathing) conditions? } \\
\text { If YES, which condition(s)? Check as many as apply. }\end{array}$ & $\begin{array}{l}\text { Yes No Not sure } \\
\text { Asthma } \\
\text { Chronic Obstructive } \\
\text { Pulmonary Disease } \\
\text { (COPD) } \\
\text { Bronchitis } \\
\text { Emphysema } \\
\text { Other } \\
\text { Please specify }\end{array}$ \\
\hline Pre-existing cardiovascular condition & $\begin{array}{l}\text { Do you have any existing cardiovascular (heart) conditions? } \\
\text { If YES, which condition(s)? Check as many as apply. }\end{array}$ & $\begin{array}{l}\text { Yes No Not sure } \\
\text { Angina } \\
\text { Previous heart } \\
\text { attack } \\
\text { Congestive heart } \\
\text { failure } \\
\text { Arrhythmia } \\
\text { Other } \\
\text { Please specify }\end{array}$ \\
\hline
\end{tabular}

health status. The final independent variable included presence of pre-existing respiratory and (or) cardiovascular conditions.

Second, qualitative questions were incorporated in the survey to give further insight into AQHI awareness, checking, following, and adoption. By focusing on knowledge/understanding of $\mathrm{AQHI}$ and reasons attributed to checking/not checking and following/not following AQHI, HBM constructs including perceived threat, benefits, barriers, cues to action and self-efficacy were identified and explained. Content analysis and descriptive codes (Hay, 2010) were used to organize the qualitative data according to the HBM constructs.

\section{Results}

\section{Sample characteristics}

Table 3 displays the characteristics of the sample including: gender, age, education, household income, area of residence, and presence of pre-existing respiratory and (or) cardiovascular conditions. The gender distribution was uneven, with $29 \%$ male and $68 \%$ female. The age range of participants was normally distributed with the greatest proportion $(23 \%)$ in the age brackets between 45 and 54; this is fairly consistent with the age distribution in Hamilton (Statistics Canada, 2013). The at- risk population representing the elderly ( $\geq 65$ years of age) accounted for $11 \%$ of the sample which is slightly lower than the 16\% (Statistics Canada, 2013) found in Hamilton. The majority of participants had a household income of $\$ 21000-\$ 50000$, whereas the minority had a household income of $\geq \$ 81000$; this is consistent with that in Hamilton (Statistics Canada, 2013). The greatest proportion of participants was high school (35\%) and college educated (33\%) which is consistent with that found in Hamilton (Statistics Canada, 2013). All of the urban and suburban areas in the city were represented. Twenty-two percent of the participants in the east lower urban area (lower SES) made up the sample; this is consistent with the population distribution in that area for Hamilton (Statistics Canada, $2012 \mathrm{~b}$ ). However, only $4 \%$ of the sample resided in the suburban areas (higher SES) of Ancaster, Dundas, and Flamborough which is much lower than the $20 \%$ that make up the population distribution in that area of Hamilton (Statistics Canada, 2012b). Within this sample, $25 \%$ reported that they have a pre-existing respiratory condition with asthma the most commonly reported; this is higher than the prevalence rate of $12.93 \%$ reported for the province of Ontario (Crighton et al., 2012). Nine percent of the participants indicated that they have a pre-existing cardiovascular condition with experiencing a heart attack as the most commonly reported; this is higher than the $5 \%$ of heart disease self reported in Ontario (Heart and Stroke Foundation, 2014). 
Table 3. Sample characteristics

\begin{tabular}{|c|c|}
\hline Characteristic & No. $(\%), n=707$ \\
\hline \multicolumn{2}{|l|}{ Gender } \\
\hline Male & $204(29)$ \\
\hline Female & $479(68)$ \\
\hline Missing & $24(3)$ \\
\hline \multicolumn{2}{|l|}{ Age (y) } \\
\hline $18-24$ & $56(8)$ \\
\hline $25-34$ & $118(17)$ \\
\hline $35-44$ & $155(22)$ \\
\hline $45-54$ & $163(23)$ \\
\hline $55-64$ & $138(19)$ \\
\hline 65 and over & $77(11)$ \\
\hline Missing & $0(0)$ \\
\hline \multicolumn{2}{|l|}{ Income } \\
\hline$<\$ 20000$ & $157(22)$ \\
\hline$\$ 21000-\$ 50000$ & $194(27)$ \\
\hline$\$ 51000-\$ 80000$ & $159(23)$ \\
\hline$\geq \$ 81000$ & $125(18)$ \\
\hline Missing & $72(10)$ \\
\hline \multicolumn{2}{|l|}{ Education } \\
\hline Elementary & $23(3)$ \\
\hline High school & $249(35)$ \\
\hline Trade & $41(41)$ \\
\hline College & $235(33)$ \\
\hline University & $154(22)$ \\
\hline Missing & $5(1)$ \\
\hline \multicolumn{2}{|l|}{ Area of residence } \\
\hline East lower & $154(22)$ \\
\hline West lower & $65(9)$ \\
\hline West upper & $135(19)$ \\
\hline East upper & $85(12)$ \\
\hline Ancaster, Dundas, and Flamborough & $28(4)$ \\
\hline Stoney Creek & $117(17)$ \\
\hline Glanbrook & $92(13)$ \\
\hline
\end{tabular}

Table 3 (continued).

\begin{tabular}{|c|c|}
\hline Characteristic & No. $(\%), n=707$ \\
\hline Missing & $31(4)$ \\
\hline \multicolumn{2}{|c|}{ Know what AQHI means } \\
\hline Yes & $244(34)$ \\
\hline No & $298(42)$ \\
\hline Not sure & $124(18)$ \\
\hline Missing & $41(6)$ \\
\hline \multicolumn{2}{|c|}{ Know where to check AQHI } \\
\hline Yes & $255(36)$ \\
\hline No & $322(46)$ \\
\hline Not sure & $95(13)$ \\
\hline Missing & $34(5)$ \\
\hline \multicolumn{2}{|c|}{ Time spent outside } \\
\hline Most & $290(41)$ \\
\hline Some & $338(48)$ \\
\hline Hardly any & $56(8)$ \\
\hline None & $4(1)$ \\
\hline Missing & $19(2)$ \\
\hline \multicolumn{2}{|c|}{ Pre-existing respiratory condition } \\
\hline Yes & $179(25)$ \\
\hline No & $525(74)$ \\
\hline Missing & $3(1)$ \\
\hline \multicolumn{2}{|c|}{ Pre-existing cardiovascular condition } \\
\hline Yes & $63(9)$ \\
\hline No & $636(90)$ \\
\hline Missing & $8(1)$ \\
\hline
\end{tabular}

\section{Quantitative data}

Table 4 presents the logistic regression results for AQHI awareness, AQHI checking, following AQHI health messages, and AQHI adoption. Each of the logistic regression results is discussed.

\section{AQHI awareness}

Sixty percent $(425 / 707)$ of participants indicated that they were aware of AQHI. Logistic regression predicting AQHI awareness identified that having a high school $(p<0.05)$ and 
Table 4. Binary logistic regression predicting AQHI awareness, checking, following, and adoption

\begin{tabular}{|c|c|c|c|c|c|c|c|c|}
\hline \multirow{2}{*}{\begin{tabular}{|l|} 
Predictor \\
\end{tabular}} & \multicolumn{2}{|c|}{ AQHI awareness } & \multicolumn{2}{|c|}{ AQHI checking } & \multicolumn{2}{|c|}{ AQHI following } & \multicolumn{2}{|c|}{ AQHI adoption } \\
\hline & B & Odds ratio & B & Odds ratio & $\mathbf{B}$ & Odds ratio & $\mathbf{B}$ & Odds ratio \\
\hline \multicolumn{9}{|l|}{ Gender } \\
\hline Male & $\begin{array}{c}\text { Reference } \\
\text { (or baseline) }\end{array}$ & & & & & & & \\
\hline Female & -0.740 & $0.477^{*}$ & 0.239 & 1.271 & 0.765 & $2.148 *$ & 0.328 & 1.389 \\
\hline \multicolumn{9}{|l|}{ Age (y) } \\
\hline $18-24$ & $\begin{array}{c}\text { Reference } \\
\text { (or baseline) }\end{array}$ & & & & & & & \\
\hline $25-34$ & 0.201 & 1.223 & 1.006 & 2.735 & -0.563 & 0.570 & 0.492 & 1.635 \\
\hline $35-44$ & 0.182 & 1.200 & 1.526 & $4.601 *$ & -0.238 & 0.789 & 1.339 & 3.815 \\
\hline $45-54$ & -0.003 & 0.997 & 2.095 & $8.129 * *$ & 0.666 & 1.947 & 1.778 & $5.917 *$ \\
\hline $55-64$ & 0.215 & 1.240 & 1.814 & $6.136^{*}$ & -0.322 & 0.725 & 0.940 & 2.561 \\
\hline$\geq 65$ & -0.418 & 0.658 & 1.420 & 4.136 & -0.445 & 0.641 & 0.135 & 1.145 \\
\hline \multicolumn{9}{|l|}{ Income } \\
\hline$<\$ 20000$ & $\begin{array}{c}\text { Reference } \\
\text { (or baseline) }\end{array}$ & & & & & & & \\
\hline$\$ 20000-\$ 50000$ & 0.024 & 1.024 & -0.268 & 0.765 & -0.177 & 0.837 & -0.074 & 0.929 \\
\hline$\$ 51000-\$ 80000$ & 0.325 & 1.384 & -0.293 & 0.746 & -0.507 & 0.602 & -0.324 & 0.723 \\
\hline$\$ 81000$ or more & 0.288 & 1.333 & -0.339 & 0.712 & -0.661 & 0.516 & -0.336 & 0.715 \\
\hline \multicolumn{9}{|l|}{ Education } \\
\hline Elementary school & $\begin{array}{c}\text { Reference } \\
\text { (or baseline) }\end{array}$ & & & & & & & \\
\hline High school & 2.521 & $12.436^{*}$ & 0.224 & 1.251 & -0.037 & 0.963 & -0.719 & 0.487 \\
\hline Trade & 0.619 & 1.858 & -0.132 & 0.876 & -1.373 & 0.253 & -1.077 & 0.341 \\
\hline College & 2.117 & $8.302 *$ & 0.583 & 1.791 & 0.082 & 1.086 & -0.283 & 0.754 \\
\hline University & 1.134 & 3.109 & 0.011 & 1.011 & 0.407 & 1.502 & -0.914 & 0.401 \\
\hline \multicolumn{9}{|l|}{ Area of residence } \\
\hline East lower & $\begin{array}{c}\text { Reference } \\
\text { (or baseline) }\end{array}$ & & & & & & & \\
\hline West lower & 0.874 & 2.396 & 0.003 & 1.003 & 1.170 & $3.221 * *$ & 0.307 & 1.359 \\
\hline West upper & 0.652 & 1.919 & -0.207 & 0.813 & 0.247 & 1.280 & -0.625 & 0.535 \\
\hline East upper & 0.593 & 1.809 & -0.434 & 0.648 & -0.264 & 0.768 & -0.764 & 0.466 \\
\hline $\begin{array}{l}\text { Ancaster/Dundas/ } \\
\text { Flamborough }\end{array}$ & 1.770 & $5.871^{*}$ & 0.184 & 1.202 & 0.879 & 2.408 & 0.587 & 1.798 \\
\hline Stoney Creek & 1.133 & $3.105^{*}$ & 0.055 & 1.057 & 0.648 & 1.913 & 0.005 & 1.005 \\
\hline Glanbrook & 0.987 & 2.683 & 0.398 & 1.489 & 0.710 & 2.035 & 0.180 & 1.198 \\
\hline
\end{tabular}


Table 4 (continued).

\begin{tabular}{|c|c|c|c|c|c|c|c|c|}
\hline \multirow{2}{*}{ Predictor } & \multicolumn{2}{|c|}{ AQHI awareness } & \multicolumn{2}{|c|}{ AQHI checking } & \multicolumn{2}{|c|}{ AQHI following } & \multicolumn{2}{|c|}{ AQHI adoption } \\
\hline & B & Odds ratio & B & Odds ratio & B & Odds ratio & B & Odds ratio \\
\hline \multicolumn{9}{|c|}{ Know what AQHI means } \\
\hline Not sure & $\begin{array}{c}\text { Reference } \\
\text { (or baseline) }\end{array}$ & & & & & & & \\
\hline Yes & 1.456 & $4.289 * *$ & 0.523 & 1.686 & 0.690 & 1.994 & 1.046 & $2.845^{*}$ \\
\hline No & -1.403 & $0.246 * * *$ & -0.214 & 0.807 & -0.777 & $0.460 *$ & -0.685 & 0.504 \\
\hline \multicolumn{9}{|c|}{ Know where to check } \\
\hline Not sure & $\begin{array}{c}\text { Reference } \\
\text { (or baseline) }\end{array}$ & & & & & & & \\
\hline Yes & 0.805 & 2.236 & 2.654 & $14.214 * * *$ & 1.314 & $3.723 * *$ & 1.835 & $6.266^{* *}$ \\
\hline No & -1.408 & $0.245^{* *}$ & -0.875 & 0.417 & -0.149 & 0.862 & -1.432 & $0.239 *$ \\
\hline \multicolumn{9}{|c|}{ Air affects health } \\
\hline Not sure & $\begin{array}{c}\text { Reference } \\
\text { (or baseline) }\end{array}$ & & & & & & & \\
\hline Yes & 0.479 & 1.615 & 0.707 & 2.027 & 0.841 & 2.318 & 0.710 & 2.034 \\
\hline No & 0.980 & 2.664 & 0.607 & 1.835 & 0.657 & 1.930 & 0.557 & 1.745 \\
\hline \multicolumn{9}{|c|}{ Length of time perceive air affects health } \\
\hline Last week & $\begin{array}{c}\text { Reference } \\
\text { (or baseline) }\end{array}$ & & & & & & & \\
\hline Last month & -1.583 & 0.205 & -3.097 & 0.045 & -2.370 & 0.093 & -17.258 & 0.000 \\
\hline Last 6 months & -4.103 & $0.017^{*}$ & -2.997 & 0.050 & -0.756 & 0.470 & 0.628 & 1.874 \\
\hline Last year & -3.111 & 0.045 & -2.195 & 0.111 & -0.348 & 0.706 & 0.076 & 1.079 \\
\hline Last 5 years & -2.975 & 0.051 & -2.241 & 0.106 & -1.166 & 0.311 & 0.094 & 1.099 \\
\hline Last 10 years & -2.736 & 0.065 & -3.332 & $0.036^{*}$ & 0.157 & 1.170 & -0.011 & 0.989 \\
\hline $\begin{array}{l}\text { More than } 10 \\
\text { years }\end{array}$ & -1.998 & 0.136 & -2.686 & 0.068 & 0.820 & 2.272 & 1.080 & 2.945 \\
\hline Other & -3.442 & 0.032 & -2.708 & 0.067 & 0.229 & 1.257 & -0.243 & 0.784 \\
\hline \multicolumn{9}{|c|}{ Physical environment affects health } \\
\hline Not sure & $\begin{array}{c}\text { Reference } \\
\text { (or baseline) }\end{array}$ & & & & & & & \\
\hline Yes & 0.209 & 1.232 & 0.081 & 1.084 & -0.155 & 0.857 & -0.341 & 0.711 \\
\hline No & -0.622 & 0.537 & 0.371 & 1.450 & -0.454 & 0.635 & -0.327 & 0.721 \\
\hline \multicolumn{9}{|c|}{ Length of time perceive physical environment affects health } \\
\hline Last week & $\begin{array}{c}\text { Reference } \\
\text { (or baseline) }\end{array}$ & & & & & & & \\
\hline Last month & -0.446 & 0.640 & 1.829 & 6.230 & 2.795 & 16.361 & -15.134 & 0.000 \\
\hline
\end{tabular}


Table 4 (continued).

\begin{tabular}{|c|c|c|c|c|c|c|c|c|}
\hline \multirow{2}{*}{ Predictor } & \multicolumn{2}{|c|}{ AQHI awareness } & \multicolumn{2}{|c|}{ AQHI checking } & \multicolumn{2}{|c|}{ AQHI following } & \multicolumn{2}{|c|}{ AQHI adoption } \\
\hline & B & Odds ratio & B & Odds ratio & B & Odds ratio & B & Odds ratio \\
\hline Last 6 months & 0.825 & 2.282 & 2.397 & 10.992 & 1.323 & 3.754 & -0.441 & 0.643 \\
\hline Last year & 1.302 & 3.675 & 1.376 & 3.960 & -0.029 & 0.971 & -1.586 & 0.205 \\
\hline Last 5 years & 0.622 & 1.863 & 2.214 & 9.149 & 0.912 & 2.489 & -0.771 & 0.463 \\
\hline Last 10 years & 0.318 & 1.375 & 2.796 & 16.376 & -0.198 & 0.820 & -0.849 & 0.428 \\
\hline $\begin{array}{l}\text { More than } \\
10 \text { years }\end{array}$ & -0.961 & 0.382 & 2.838 & 17.087 & -0.557 & 0.573 & -1.763 & 0.171 \\
\hline Other & 1.618 & 5.044 & 2.373 & 10.734 & -2.919 & 0.054 & -2.600 & 0.074 \\
\hline \multicolumn{9}{|l|}{ Time spent outside } \\
\hline None & $\begin{array}{l}\text { Reference } \\
\text { (or baseline) }\end{array}$ & & & & & & & \\
\hline Most & 0.542 & 1.720 & -0.074 & 0.928 & -0.376 & 0.687 & -0.794 & 0.452 \\
\hline Some & 1.475 & 4.369 & -0.173 & 0.841 & 0.353 & 1.424 & -0.800 & 0.449 \\
\hline Hardly any & 1.997 & 7.369 & -1.318 & 0.268 & -0.318 & 0.728 & -1.348 & 0.260 \\
\hline \multicolumn{9}{|c|}{ Health status (self-reported) } \\
\hline Very good/good & $\begin{array}{c}\text { Reference } \\
\text { (or baseline) }\end{array}$ & & & & & & & \\
\hline $\begin{array}{l}\text { Fair/poor/very } \\
\text { poor }\end{array}$ & -0.214 & 0.807 & 0.447 & 1.564 & 0.130 & 1.139 & 0.128 & 1.137 \\
\hline \multicolumn{9}{|c|}{ Pre-existing respiratory condition } \\
\hline Yes & 0.488 & 1.630 & 0.508 & 1.662 & -0.337 & 0.714 & 0.051 & 1.052 \\
\hline No & $\begin{array}{l}\text { Reference } \\
\text { (or baseline) }\end{array}$ & & & & & & & \\
\hline \multicolumn{9}{|c|}{ Pre-existing cardiovascular condition } \\
\hline Yes & 0.695 & 2.003 & -0.411 & 0.663 & 0.594 & 1.811 & -0.203 & 0.816 \\
\hline No & $\begin{array}{l}\text { Reference } \\
\text { (or baseline) }\end{array}$ & & & & & & & \\
\hline Cox and Snell $R^{2}$ & \multicolumn{2}{|c|}{0.416} & \multicolumn{2}{|c|}{0.395} & \multicolumn{2}{|c|}{0.316} & \multicolumn{2}{|c|}{0.357} \\
\hline Nagelkerke $R^{2}$ & \multicolumn{2}{|c|}{0.573} & \multicolumn{2}{|c|}{0.560} & \multicolumn{2}{|c|}{0.422} & \multicolumn{2}{|c|}{0.543} \\
\hline
\end{tabular}

Significance levels: ${ }^{*} p<0.05,{ }^{* *} p<0.01,{ }^{* *} p<0.001$. B is the coefficient on the independent variable.

(or) college education $(p<0.05)$; living in the suburban areas of Ancaster, Dundas, and Flamborough $(p<0.05)$ and Stoney Creek $(p<0.05)$ (higher SES areas); and knowing where to check the AQHI $(p<0.01)$ are positively associated with being aware of AQHI. Conversely, being female $(p<0.05)$, perceiving neighbourhood air as affecting health for the last 6 months $(p<0.05)$, and not knowing or having an understanding of what AQHI means $(p<0.001)$ are negatively associated with the probability of being aware of AQHI.

\section{AQHI checking}

Thirty-six percent $(256 / 707)$ of participants reported that they know where to check for the AQHI, whereas only $27 \%$ (190/ 707) reported that they check AQHI. Of those who check AQHI, 27\% (52/190) reported that they always check, 43\% $(81 / 190)$ reported that they check half the time, and $8 \%(16 /$ $190)$ reported that they rarely check. Logistic regression predicting AQHI checking identified that being 35-44 ( $p<0.05)$, 
45-54 $(p<0.01)$, and 55-64 $(p<0.05)$ years of age and knowing where to check $(p<0.001)$ the AQHI are positively associated with the probability of checking AQHI numbers. However, perceiving neighbourhood air as affecting health for the last 10 years is negatively associated with the probability of checking AQHI numbers $(p<0.05)$.

\section{AQHI following health messages}

Forty-three percent $(303 / 707)$ of all participants reported that they follow AQHI health messages. However, 37\% (113/303) of those who reported following AQHI health messages were not checking AQHI numbers; therefore, these individuals are relying on cues other than AQHI to implement health protective behaviours. Logistic regression results indicate that being female $(p<0.05)$, having an understanding of what the AQHI means $(p<0.05)$, knowing where to check AQHI numbers $(p<0.05)$, and residing in the west lower area $(p<0.05)$ of the city are positively associated with the probability of following AQHI health messages.

\section{AQHI adoption}

Twenty percent $(142 / 707)$ of the participants were aware of $\mathrm{AQHI}$, check AQHI, and follow AQHI health messages, and therefore have adopted AQHI. Within the at-risk population ( $\geq 65$ years and those with pre-existing respiratory and (or) cardiovascular conditions), 79\% (253/319) have not adopted the AQHI. Logistic regression predicting AQHI adoption identified that being $45-54$ years of age $(p<0.05)$, having an understanding of the AQHI means $(p<0.05)$, and knowing where to check AQHI numbers $(p<0.01)$ are positively associated with the probability of adopting AQHI.

\section{Qualitative data}

The qualitative data collected from 707 surveys is presented with the quantitative questions they were designed to expand upon.

\section{Knowledge/understanding of AQHI}

To expand upon the quantitative question asking: "Do you know what a High AQHI (7-10) means?" participants were asked to explain what it means to them. Eighty-four percent of participants responded to this open-ended question by expanding on aspects of limiting outdoor activity, identifying at-risk populations and adverse health effects due to air pollution exposure. Participants explained that High AQHI (7-10) means: "One should take protective measures or limit outdoor activities" and "People with respiratory and other conditions impacted by high level." Participants explained adverse impacts on health and outdoor activity by indicating that: "It means I may have trouble enjoying activities outdoors because of breathing issues", and "That your breathing could be affected especially if you have breathing problems."

Although responses were consistent with the purpose of $\mathrm{AQHI}$, confusion in messaging between the AQI and AQHI was apparent. Participants indicated that they believed high AQHI means: "Smog alert is out" and that "A health warning goes out to the media newspaper, radio, TV, and internet to warn people with health conditions esp[cially] asthmatics and seniors." Unlike with the AQI, smog alerts and media advisories were not issued with the AQHI.

To further explore knowledge/understanding of AQHI and expand upon the quantitative question: "Do you know where to check for daily Air Quality Health Index (AQHI)?" participants were asked to explain "where" they check AQHI. Participants explained that they check the AQHI on the television, radio, websites, and in the newspaper. Specifically, the "Weather Network," local television news, and local newspapers were named as sources for AQHI information. Currently, the local news channel and the local newspaper do not post AQHI information in the city of Hamilton. Therefore, although participants were able to explain the purpose of the AQHI, there appears to be confusion between the AQI and AQHI, as well as where to find AQHI information.

\section{Reasons attributed to checking/not checking AQHI and following/not following AQHI health messages}

With the intention to further expand upon the quantitative question: "Do you check the Air Quality Health Index (AQHI)?" participants were asked to explain "why or why not?" Likewise, to further expand upon the quantitative question: "Do you follow AQHI Health Messages which tell you when to consider reducing or re-scheduling outdoor physical activity?" participants were asked to explain "why or why not?"

Participants who checked AQHI and follow AQHI health messages explained that they perceived the benefits of checking and following health messages as those related to health protection for self and those they care for via familial and (or) occupational duties. Participants indicated that they follow AQHI health messages because they want to ensure: "safety for kids" and because they "work with children, so I really need to be responsible of health and safety of myself and others."

Consistently, participants identified lack of knowledge as a perceived barrier to checking and following AQHI health messages. Participants indicated that they are not able to check and follow AQHI health messages because they: "Don't know where." An additional barrier to checking and following health messages was lack of time. Participants explained that they: "Don't always have time" and are "Too busy with childrenthe index won't really influence my activities." Moreover, participants described issues pertaining to self-efficacy as a reason for not checking and following AQHI health messages by responding: "Don't know how."

Reasons for not checking and following health messages varied among lower and higher SES participants. Those in the east lower area (lower SES) indicated that they do not check and follow AQHI health messages since they: "cannot control it" and "cannot change it." This suggests that issues concerning empowerment should be explored. Although those in the 
suburban areas (higher SES) of Ancaster, Dundas, Flamborough, Stoney Creek, and Glanbrook indicated that checking and following AQHI health messages is: "not a high priority" and "not too much of an issue in the country over the escarpment." This suggests that issues around optimism bias should be explored.

In addition, participants indicated that they do not check and follow AQHI health messages because they rely on sensory cues that they can "visually see and hear." Moreover, they indicated that they do not check and follow AQHI health messages because they "just go by self smarts" and "Sometimes - don't really need to-can tell by way air looks." Another participant indicated that checking AQHI is not necessary because: "I use the temperature to determine." Moreover, reliance on media advisories as a cue to modify health behaviour was provided as a reason for not checking and following health messages. Participants indicated that "If on news" and "Only if mentioned on radio" they would implement health protective behaviors.

\section{Discussion}

Reasons for checking and following AQHI included acknowledgement of the perceived threat of adverse health effects from exposure to air pollution and the perceived benefits of health protection for self and those cared for via familial and (or) occupational duties. Barriers to checking and following health messages included lack of knowledge and time and reliance on sensory cues and media advisories. Reasons for not checking and following health messages varied between lower and higher SES groups. Moreover, self-efficacy was apparent as a reason for not checking and following AQHI health messages.

As the HBM and previous research (Elliott et al., 1999; Eyles et al., 2009; Wilson et al., 2009; Simone et al., 2012) proposed, demographics (gender, age, education, and area of residence), knowledge/understanding, and individual perceptions (neighborhood air effects on health) were significant predictors of AQHI awareness, checking, following health messages, and adoption in this study. The qualitative data helped explain these predictors and guides intervention strategies to increase AQHI adoption.

The findings suggest that intervention strategies must account for gender differences in awareness and following health messages. This study's findings are consistent with a US study on the Air Quality Index (AQI) and awareness which found that women were less likely to be aware than men (Johnson, 2012). Thus, the intervention strategy should focus on making women aware through promotional channels (i.e., women's health/fitness magazines).

Although females were less aware of the AQHI than males, they were more likely than males to follow AQHI health messages. Researchers who examined sun protective behaviour also found that females were more likely to engage in the health protective behaviour than males (Buller et al., 2011), as did researchers who examined health protective behaviors in response to West Nile virus (Elliott et al., 2008). Therefore, an intervention strategy should focus on encouraging males to reduce or reschedule outdoor physical activity according to the AQHI; this messaging could be done with the assistance of health professionals as studies have found that people are more likely to implement health protective behaviors in response to poor air quality when informed by health professionals (Wen et al., 2009).

In addition, intervention strategies must focus on increasing knowledge/understanding of AQHI. As Elliott et al. (1999) found when examining AQI awareness in Hamilton, recognizing the term "AQHI" does not necessarily indicate knowledge/ understanding of what it means. Accordingly, the intervention strategy must clearly define the purpose of the AQHI; this will address confusion between AQI and AQHI. The need to increase knowledge/understanding is further supported by the fact that being at risk ( $\geq 65$ years of age and having a preexisting respiratory and (or) cardiovascular condition) was not found to be a significant predictor of AQHI adoption; participants belonging to the at-risk population in this study did not perceive severity nor believe that they may be more sensitive to air pollution than the general population. Therefore, intervention strategies must clearly define the at-risk populations such that they are able to self-identify and understand that they are considered at risk. Moreover, increasing the public's knowledge/understanding with respect to finding and using AQHI numbers to address self-efficacy issues must be considered.

Furthermore, interventions strategies must account for variations in environmental risk perceptions found in lower and higher SES areas of the city (Elliott et al., 1999; Eyles et al., 2009; Wilson et al., 2009; Simone et al., 2012). Differences in perceived threat and AQHI awareness have been identified as reasons for not adopting AQHI. As other studies have found with AQI (Johnson, 2012), this study has found a higher level of AQHI awareness among individuals of higher SES. Moreover, the data suggest that individuals who perceive their neighborhood air impacting their health for an extended period of time (10 years) may rely on sensory cues (see, smell, feel) to implement health protective behaviors as opposed to AQHI numbers. This is consistent with other studies that found sensory cues prevail over AQI (Bickerstaff and Walker, 2001; Bush et al., 2001). This study suggests that issues concerning empowerment are important in understanding why individuals in lower SES are not adopting AQHI, whereas issues concerning optimism bias are important in understanding why individuals in higher SES are not adopting AQHI.

The finding that individuals in the west lower area were more likely to follow AQHI health messages than those in the east lower area supports community engagement as a successful approach to health promotion and community empowerment (Milton et al., 2011). The west lower area of the city has been engaged in different environmental and health promoting initiatives such as the "Bike Share" initiative (City of Hamilton, 2014).

To address neighbourhood area variations in perceived threat and AQHI awareness, the intervention strategies should provide AQHI information at a neighborhood scale. By providing $\mathrm{AQHI}$ information that represents conditions within the public's immediate environment, issues of empowerment in 
the lower SES, and optimism bias in the higher SES can be addressed.

Finally, local AQHI media advisories should be incorporated as participants indicated that they rely on media advisories as cues to implement health protective behaviours. The AQHI was designed as a self-calibration tool such that individuals could determine what level is a detriment to their individual health. As such, this varies between individuals. Taking individual variability into account and applying the precautionary principle, AQHI (7-10) High Health Risk levels at which it is recommended that the at-risk population "reduce or reschedule strenuous" (Environment Canada, 2013) outdoor activity could be proposed as a level for which public media advisories are provided.

\section{Limitations}

Although our convenience sample was fairly representative with respect to distribution of age, income, education, and population according to city divisions (Statistics Canada, 2012b, 2013), overrepresentation of females and underrepresentation in higher SES suburban areas may have contributed to self-selection sampling, which may have impacted results. Additionally, as with all studies using surveys, recall and response bias may impact the results. Moreover, even though the qualitative questions encouraged participants to expand upon responses instead of simply agreeing, acquiescence bias may impact the results. Another limitation includes the HBM not accounting for emotional aspects of behaviour (Glanz et al., 2008) and impacts of past behaviours (habits) as predictors of future behaviours (Ouellette and Wood, 1998).

\section{Conclusion}

As HBM posits, once AQHI knowledge is gained, perceived susceptibility, perceived severity, perceived benefits, perceived barriers, cues to action, and self-efficacy need to be considered with respect to AQHI adoption. Increasing AQHI knowledge is critical for AQHI adoption to occur. Particular focus on increasing AQHI knowledge should be given to females in lower SES areas

Accordingly, with respect to perceived threat (perceived susceptibility and severity), risk to health from outdoor air pollution exposure must be clearly communicated such that the benefit of using the AQHI to decrease the potential for adverse health effect outweighs the barrier of time commitment required to check AQHI and follow AQHI health messages. Particular attention should be given to at-risk populations so that they are able to accurately perceive threat to health from exposure to air pollution and implement health protective behaviour accordingly. Additionally, attention to increasing the likelihood of males following AQHI health messages should to be considered.

These intervention strategies account for AQHI adoption at the individual level. Intervention strategies that examine AQHI adoption at the organizational and community levels and consider empowerment, community engagement, and optimism bias are recommended to develop a comprehensive public health approach to increase AQHI adoption (Glanz et al., 2008).

\section{References}

Barakat-Haddad, C., Elliot, S., and Pengelly, D. 2013. Childhood exposure to air pollution as a potential contributor of chronic nonrespiratory inflammatory disorders: A longitudinal prospective cohort study in Hamilton. Canada. J. Environ. Protec, 4: 779-788. doi: 10.4236/jep.2013.48091.

Bickerstaff, K., and Walker, G. 2001. Public understandings of air pollution: The "localisation" of environmental risk. Global Environ. Change, 11: 133-145. doi: 10.1016/S0959-3780(00)00063-7.

Buller, D., Cokkinides, V., Hall, I., Hartman, A., Saraiya, M., Miller, E., et al. 2011. Prevalence of sunburn, sun protection, and indoor tanning behaviors among Americans: Review from national surveys and case studies in 3 states. Am. Acad. Dermatol, 65(5): S114-S123.

Bush, J., Moffatt, S., and Dunn, C.E. 2001. Even the birds around here cough: Stigma, air pollution and health in Teesside. Health Place, 7: 47-56. doi: 10.1016/S1353-8292(00)00037-X.

Buzzelli, M., Jerrett, M., Burnett, R., and Finkelstein, N. 2003. Spatiotemporal perspectives on air pollution and environmental justice in Hamilton, Canada, 1985-1996. Ann. Assoc. Am. Geogr, 93(3): 557-573. doi: 10.1111/1467-8306.9303003.

City of Hamilton. 2014. Hamilton Bike Share. Available at http:// hamiltonbikeshare.org/ [accessed 8 August 2014].

Crighton, E., Feng, J., Gershon, A., Guan, J., and To, T. 2012. A spatial analysis of asthma prevalence in Ontario. Can. J. Public Health, 103(5): e384-e389.

DeLuca, P., Buist, S., and Johnston, N. 2012. The code red project: Engaging communities in health system change in Hamilton, Canada. Soc. Indic. Res, 108: 317-327. doi: 10.1007/s11205-012-0068-y.

Dockery, D., Pope, C., Xu, X., Spengler, J., Ware, J., Fay, M.E., et al. 1993. An association between air pollution and mortality in six U.S. cities. N. Engl. J. Med, 329(24): 1753-1759. doi: 10.1056/ NEJM199312093292401.

Elliott, S.J., Cole, D.C., Krueger, P., Voorberg, N., and Wakefield, S. 1999. The power of perception: Health risk attributed to air pollution in an urban industrial neighbourhood. Risk Anal, 19: 621-633.

Elliott, S.J., Loeb, M., Harrington, D., and Eyles, J. 2008. Heeding the message? Determinants of risk behaviours for West Nile Virus. Can. J. Public Health, 99(2): 137-141.

Environment Canada AQHI. 2013. Available at http://www.ec.gc.ca/ cas-aqhi/ [accessed 7 August 2014].

Eyles, J., Wilson, K., Mu, L., Keller-Olaman, S., and Elliot, S. 2009. What people think about the environment and its relationship to their health: perceptions of health at different scales of environment in Hamilton, Ontario. Local Environ, 14(10): 981-998. doi: $10.1080 / 13549830903277409$.

Glanz, K., Rimer, B. and Viswanath, K. 2008. Health behaviour and health education: theory, research, and practice. 4th ed. San Francisco, CA: Jossey-Bass.

Hay, I. 2010. Qualitative research methods in human geography. 3rd ed. Oxford: Oxford University Press (Don Mills, ON: CND).

Heart and Stroke Foundation. 2014. Statistics. Available at http:// www.heartandstroke.on.ca/site/c.pvI3IeNWJwE/b.3581729/k.359A/ Statistics.htm [accessed 7 August 2014]. 
Hochbaum, G. 1958. Public participation in medical screening programs: A socio-psychological study. Washington, DC: US Department of Health, Education, and Welfare.

International Agency for Research on Cancer (IARC), World Health Organization (WHO). 2013. The carcinogenicity of outdoor air pollution. Lancet Oncol. 14(13): 1262-1263. doi: 10.1016/S14702045(13)70487-X

Jerrett, M., Burnett, R.T., Kanaroglou, P., Eyles, J., Finkelstein, N., Giovis, C., et al. 2001. A GIS-environmental justice analysis of particulate air pollution in Hamilton, Canada. Environ. Plan. A, 33: 955-973. doi: 10.1068/a33137.

Johnson, B. 2012. Experience with urban air pollution in Paterson, New Jersey and implications for air pollution communication. Risk Anal, 32(1): 39-53. doi: 10.1111/j.1539-6924.2011.01669.x.

Kerigan, A., Goldsmith, C., and Pengelly, D. 1986. A three-year cohort study of the role of environmental factors in the respiratory health of children in Hamilton, Ontario. Am. Rev. Respir. Dis, 133(6): 987-993.

Legler, J., Meissner, H.I., Coyne, C., Breen, N., Chollette, V., and Rimer, B.K. 2002. The effectiveness of interventions to promote mammography among women with historically lower rates of screening. Cancer Epidemiol. Biomark. Prev, 11: 59-71.

Milton, B., Attree, P., French, B., Povall, S., Whitehead, M., and Popay, J. 2011. The impact of community engagement on health and social outcomes: a systematic review. Community Dev. J, 47(3): 316-334. doi: 10.1093/cdj/bsr043.

Noar, S.M., Black, H.G., and Pierce, L.B. 2009. Efficacy of computer technology-based HIV prevention interventions: a meta-analysis. AIDS, 23: 107-115. doi: 10.1097/QAD.0b013e32831c5500.

Ouellette, J.A., and Wood, W. 1998. Habit and intention in everyday life: The multiple processes by which past behavior predicts future behavior. Psychol. Bull, 124(1): 54-74. doi: 10.1037/0033-2909. 124.1.54.

Pope, C., Burnett, R., Thun, M., Calle, E., Krewski, D., Ito, K., et al. 2002. Lung cancer, cardiopulmonary mortality, and long-term exposure to fine particulate air pollution. JAMA, 287: 1132-1141. doi: $10.1001 /$ jama.287.9.1132.

Rosenstock, I. 1974. The health belief model and preventative health behavior. Health Educ. Monogr, 2(4): 354-386. doi: 10.1177/ 109019817400200405.

Semenza, J., Wilson, D., Parra, J., Bontempo, B., Hart, M., Sailor, D., et al. 2008. Public perception and behavior change in relationship to hot weather and air pollution. Environ. Res, 107: 401-411. doi: 10.1016/j.envres.2008.03.005.

Simone, D., Eyles, J., Newbold, K.B., Kitchen, P., and Williams, A. 2012. Air quality in Hamilton: Who is concerned? Perceptions from three neighbourhoods. Soc. Indic. Res, 108: 239-255. doi: $10.1007 / \mathrm{s} 11205-012-0064-2$.

Statistics Canada. 2012a. Focus on geography series, 2011 Census. Statistics Canada Catalogue no. 98-310-XWE2011004. Ottawa, ON: Statistics Canada. Analytical products, 2011 Census. Last updated October 24, 2012.

Statistics Canada. 2012b. 2001-2011 Census population and dwelling counts for the city of Hamilton by Ward. Available at http://map. hamilton.ca/static/pdfs/wardmaps/AllWards_Statistics.pdf. [accessed 17 January 2016].

Statistics Canada. 2013. Hamilton, C, Ontario (Code 3525005) (table). National Household Survey (NHS) profile. 2011 National Household Survey. Statistics Canada Catalogue no. 99-004-XWE. Ottawa. Released September 11, 2013.

Stieb, D.M., Paola, J., and Neuman, K. 1996. Do smog advisories work? Results of an evaluation of the Canadian smog advisory program. Can. J. Public Health, 87(3): 166-169.

Trifiletti, L.B., Gielen, A.C., Sleet, D.A., and Hopkins, K. 2005. Behavioral and social sciences theories and models: Are they used in unintentional injury prevention research? Health Educ. Res, 20: 298-307. doi: 10.1093/her/cyg126.

Wallace, J., Corr, D., and Kanaroglou, P. 2010. Topographic and spatial impacts of temperature inversions on air quality using mobile air pollution surveys. Sci. Total Environ, 21: 5086-5098. doi: 10.1016/j.scitotenv.2010.06.020.

Wen, X., Balluz, L., and Mokdad, A. 2009. Association between media alerts of air quality index and change of outdoor activity among adult asthma in six states, BRFSS, 2005. J. Community Health, 34: 40-46. doi: 10.1007/s10900-008-9126-4.

WHO. 2014. Burden of disease from Ambient Air Pollution for 2012. Summary results. Geneva: World Health Organization.

Wilson, K., Eyles, J., Elliott, S., and Keller-Olaman, S. 2009. Health in Hamilton neighbourhoods. Health Place, 15: 374-382. doi: 10.1016/j.healthplace.2008.07.002.

Zanobetti, A., and Schwartz, J. 2009. The effect of fine and coarse particulate air pollution on mortality: A national analysis. Environ. Health Perspect, 117: 898-903. doi: 10.1289/ehp.0800108. 